\title{
Negative-Pressure Wound Therapy Versus Advanced Moist Wound Therapy in the Treatment of Diabetic Foot Wounds
}

\author{
Ahmed Hussieny El-Barbary, MD; Hasan Abdel-Aty, MD. \\ Unit of Vascular Surgery, General Surgery Department, Faculty of Medicine, \\ Tanta University, Egypt
}

\begin{abstract}
Aim of the work: To evaluate clinical effectiveness and safety of negative pressure wound therapy (NPWT) compared with advanced moist wound therapy (AMWT) (mainly hydrogels, tulle and alginates) for treatment of diabetic foot wounds.

Patients and methods: This was a prospective randomized study conducted on (40) patients with chronic diabetic foot wounds who were divided into (NPWT) group through vacuum assisted closure $(V A C)^{\circledR}$ System and (AMWT) group mainly hydrocolloids, tulle or alginates. The study evaluated treatment until day (112) or ulcer closure by any means. The primary end point was incidence and median time of complete wound closure by either surgery or secondary intention. Wound assessment entailed wound area, progress of granulation tissue, infection, need for debridement, discharge and pain.
\end{abstract}

Results: A significantly greater percentage of (NPWT) wounds (60\%, 12 of 20) achieved complete closure than (AMWT) wounds (30\%, 6 of 20) (P=0.033). Median time to complete wound closure was (76 days) for (NPWT) and (91 days) for (AMWT) $(P=0.024)$. Median time to (76-100\%) granulation was significantly shorter in (NPWT) (50 days) than in AMWT patients (88 days) $(P=0.0001)$.

Conclusion: NPWT is as safe as and more efficacious than AMWT for the treatment of diabetic foot wounds.

Key words: Negative pressure- diabetic foot- moist dressing-granulation.

\section{Introduction:}

Wound repair is an orchestra of highly integrated biological and molecular events. ${ }^{1}$ These events entail debris removal, infection control, clearance of inflammation, angiogenesis, granulation tissue formation, contraction, connective tissue matrix remodeling and maturation. ${ }^{2}$ Certain pathophysiologic and metabolic conditions can alter this sequence so that healing is impaired or delayed, resulting in chronic, nonhealing wounds. ${ }^{3,4}$

Diabetic foot wounds readily become chronic, and the factors that delay wound healing are multiple and relate both to diabetes and its complications which include ischaemia, neuropathy and impaired immunity against infection. 4,5

The optimal treatment for diabetic foot wounds remains contradictory. Salinemoistened gauze has been the standard dressing; however, it has been difficult to continuously maintain a moist wound environment. ${ }^{6}$ This has led to the development of various advanced moist wound therapy (AMWT) dressings such as hydrocolloid, tulle, hydrogels, foams and alginates which provided more consistent moisture retention. Hyperbaric oxygen therapy and bioengineered skin substitutes are other methods that have been used. The significant expense of these methods as well as the lack of sufficient evidence to recommend their efficacy are triggering researchers to search for an optimum therapy. ${ }^{7}$

Negative pressure wound therapy (NPWT) is a newer noninvasive adjunctive therapy system that uses controlled negative pressure 
using Vacuum-Assisted Closure device $(\mathrm{VAC})^{\circledR}$ to help promote wound healing by removing discharge from open wounds through a sealed dressing and tubing which is connected to a collection canister. The use of sub-atmospheric pressure dressings, available commercially as a VAC device, has been shown to be an effective way to accelerate healing of various wounds. ${ }^{8-11}$

\section{Aim of the work:}

This was a prospective randomized study evaluating the clinical effectiveness and safety of NPWT compared with AMWT (mainly hydrogels, tulle and alginates) for the treatment of diabetic foot wounds.

\section{Patients and methods:}

Study design: From February 2012 to March 2014 a prospective study was conducted on 40 patients with chronic diabetic foot wounds at Vascular Surgery Unit, Tanta University Hospitals. The eligible patients were divided equally and randomly into, a study group (NPWT) subjected to negative pressure wound therapy through Vacuum Assisted Closure (VAC) ${ }^{\circledR}$ System and a control group (AMWT) subjected to once daily change of advanced moist wound therapy dressing (hydrocolloids, tulle or alginate).

Inclusion criteria: Chronic diabetic foot wounds with the following criteria:

- Diabetic adults $\geq 18$ years with diabetic foot wounds dating for a minimum of 4 weeks corresponding to University of Texas grades 2 or 3(stages A or B) Table (1) and $\geq 4 \mathrm{~cm} 2$ in area after debridement.

- Adequate blood perfusion of the affected leg: Assessed by a transcutaneous oxygen tension $\geq 30 \mathrm{mmHg}$, ankle brachial index value $\geq 0.7$ with toe pressure $\geq 30$ $\mathrm{mmHg}$ or Doppler ankle arterial waveforms that are triphasic or biphasic.

Exclusion criteria:

- Wounds with malignant disease or untreated osteomyelitis .

- Wounds resulting from venous insufficiency.

- Wounds treated by hyperbaric oxygen, skin substitutes, growth factor products or enzymatic debridement within 30-days of enrollment.

- Patients being treated with corticosteroids, immunosuppressive drugs, anticoagulation therapy or chemotherapy.

- Patients with inadequate lower extremity perfusion.

- Patients with active Charcot disease or collagen vascular disease.

- Patients having allergy to any disposal component of each treatment arm.

- Patients having serious preexisting cardiovascular, pulmonary and immunological disease.

- Pregnant and nursing mothers.

- Indication for amputation above the ankle level of the same extremity.

Randomization: It was accomplished by generating blocks of numbers through http:// www.randomizer.org. Numbers were assigned to a treatment group and sealed in opaque sequentially numbered envelopes containing a paper labeled with treatment and patient hospital number. At patient randomization, treatment was assigned on the basis of the next sequentially labeled envelope. Due to physical differences between the treatment regimens it is not possible to blind either participant or physician to the treatment arm after random assignment.

Outcome variables: The reported data included; patients' demographic data, diabetic history and control through (HbAlc) estimation and presence of comorbidities. Description of wounds included their location, measurements, presence of exudates and percentage of granulation tissue. Wound cultures were sent before application of dressings and weekly thereafter. Plain-x-ray and duplex study were done for exclusion of osteomyelitis and ischemic etiology.

Pre-enrollment measures: Prior to enrollment in the study, aggressive debridement was performed on all wounds in the operating theatre. Debridement entailed the excision of all necrotic and infected tissue until healthy bleeding tissue was reached. After debridement, the wound was thoroughly cleansed and irrigated by saline Figure (1). 
Setting of treatment method: Study therapy was started in-hospital or at home and continued at home whenever possible. Study therapy was continued until day 112 (16 weeks) or wound closure by any means.

Methods of application of the dressing:

VAC dressings: The (NPWT) system used in this study was Vacuum-Assisted Closure $\quad\left(\right.$ VAC) ${ }^{\circledR}$ device; $\quad$ (VENTURI ${ }^{\circledR}$ NPWT System) (Talley medical Group Ltd. Abbey Park Industrial Estate, Romsey, Hampshire, SO519DQ England). VAC ${ }^{\circledR}$ System includes an open cell polymer foam dressing cut to conform to the wound bed shape, a transparent film sheet used to seal the dressing, this covering sheet is connected to a plastic drainage tube which in turn is attached to a fluid collection reservoir and a vacuum pump. This pump provides intermittent or continuous pressure ranging from $(-25 \mathrm{mmHg})$ through $(-200 \mathrm{mmHg})$. The negative pressure used ranged from $(-40$ to $-125 \mathrm{mmHg}$ ). Intermittent pressure was used post-debridement and in oozing wounds as 5 minutes "on" and 2 minutes "off". Lower negative pressures were used if patient felt pain or much bleeding was observed. NPWT dressings were changed every $48-72$ hours.

Advanced Moist Wound Therapy (AMWT): The control group received once daily dressing change using advanced moist wound dressings (hydrocolloid gels, tulle or alginates).

Study end-points: The primary end point was the incidence and median time of complete wound closure by either surgery or secondary intention. Secondary end points included the incidence and median time until optimal preparation of the wound bed for further treatment ( $76-100 \%$ granulation) and a reduction in complications, including secondary amputations.

Surgical interventions such as wound suture, skin grafting or flaps were allowed only if the wound bed was adequately prepared. Sustainment of complete wound closure had to be proven 14 days after the intervention.

Patients were examined weekly for the first 4 weeks (day 28) then every other week until day (112) or wound closure by any means. At each visit, wounds were assessed for wound area, progress of granulation tissue formation, presence of infection, need for further debridement, amount of discharge and pain evaluated via pain visual analogue scale (scale from 1-100). Standard broad spectrum antibiotics were administered to all patients initially and later according to the culture sensitivity report.

Definitions: Complete wound healing was defined as skin closure $(100 \%$ reepithelization) without drainage or dressing requirements.

Wound size was calculated as described by Xakellis and Frantz. ${ }^{13}$

Wound surface area $=$ Length $\times$ Width $\times 0.783$. $(0.783$ is $\Pi / 4)$.

Statistical analysis: Continuous demographic variables (age, sex, and comorbidities) were summarized as descriptive statistics (number and mean \pm SD), and 95\% two-sided CIs and compared between groups with a two-sample $t$ test. Incidence and time of complete wound closure, and duration of wound bed preparation were compared using the Kaplan-Meier survival analysis. The log-rank test was used to test for statistically significant differences between groups using GraphPad Prism ${ }^{\circledR} 6$.

Ethical issue :Written informed consent was obtained from all patients. The study was approved from the Faculty ethical committee.

\section{Results:}

Patients' Characteristics: Patients and wounds characteristics of both groups are shown in Table (2). The mean age of patients in (NPWT) group was $(55.2 \pm 7.8)$ years and in (AMWT) group was $(53.50 \pm 8.1)$ years. The age distribution was comparable and statistically insignificant in both groups (P $=0.13)$. In (NPWT) group, 11/20 (55\%) of the patients were males whereas in (AMWT) group, $13 / 20(65 \%)$ of the patients were males. Wounds in (NPWT) group had a mean duration of $(7.9 \pm 3)$ weeks and a range of $(4-16)$ weeks while in (AMWT) group mean wounds duration was $(8 \pm 3.13)$ weeks with a range of $(4-14)$ weeks. 
The randomization method resulted in an even distribution of characteristics between treatment groups. Although the mean wounds surface area was larger in the (NPWT) group, no significant statistical differences were observed in common risk factors known to be associated with delayed wound healing Table (2).

Wound Exudates: At first week it was observed that all patients in both groups had exudates from the wound. The discharge kept on decreasing over the period of observation in both groups; however, (NPWT) patients had a faster rate of disappearance of exudates. By the end of $8^{\text {th }}$ week, wound exudates were present in only (15\%) of NPWT as compared to $(35 \%)$ in (AMWT) patients; $(\mathrm{P}=0.12)$.

Additional Debridement: Along with the pretreatment surgical debridement performed for all included patients, no additional surgical debridement was performed in 16 patients during the course of (VAC therapy); whereas 4 patients required an additional surgical debridement. In (AMWT) group, 12 patients needed no additional surgical debridement, 7 patients needed an additional surgical debridement and one patient required 2 additional surgical debridements.

Wound Closure Analysis: Analysis demonstrated that a significantly greater percentage of NPWT-treated wounds $(60 \%$, 12 of 20) achieved complete closure than AMWT-treated wounds $(30 \%, 6$ of 20$)$; $(\mathrm{P}=0.033)$. Kaplan-Meier median time to complete wound closure Figure (2) was (76 days) (95\% CI $1.11-7.21)$ for (NPWT) and (91 days) (95\% CI $0.14-0.89)$ for (AMWT) ( $\mathrm{P}=0.024)$.

Again, significantly more (NPWT) patients (16 of 20, 80\%) achieved $75 \%$ wound closure than (AMWT) patients (12 of 20,60\%); (P $=0.036)$. Kaplan-Meier median estimates for $75 \%$ wound closure times were (63 days) (95\% CI $1.09-5.03)$ for (NPWT) and (83 days) (95\% CI $0.19-0.91)$ for (AMWT); (P $=0.015$ ).

In assessing wound area, a significant reduction was observed from baseline values by the end of $4^{\text {th }}$ week in (NPWT) patients $\left(-6.2 \mathrm{~cm}^{2}, 23.4 \%\right)$ than (AMWT) patients $\left(-3.9 \mathrm{~cm}^{2}, 16.25 \%\right) ; \quad(\mathrm{P}=0.021)$. Also, a significant total wound area reduction was observed in (NPWT) patients $\left(-22.9 \mathrm{~cm}^{2}, 86.4 \%\right)$ than in (AMWT) patients $\left(-18.18 \mathrm{~cm}^{2}, 75.7 \%\right)$ at the end of study (day $-112) ;(\mathrm{P}=0.002)$. After sufficient wound bed preparation, $(30 \%, 6$ of 20) NPWT-treated wounds and $(25 \%, 5$ of 20$)$ AMWT-treated wounds were surgically closed by split thickness skin grafts, flaps or sutures.

Granulation Tissue: At the baseline evaluation of wounds, it was observed that 10 patients $(50 \%)$ presented by $(0-10 \%)$ granulation tissue in (NPWT) group versus 9 patients (45\%) in (AMWT) group. With ongoing therapy, all these (NPWT) patients $(100 \%)$ and 6 of $9(66.7 \%)$ (AMWT) patients achieved (76-100\%) granulation tissue formation; $(\mathrm{P}=0.025)$. For the whole study population, the median time needed to achieve (76-100\%) granulation tissue formation Figure $(3,4)$ was significantly shorter in (NPWT) patients (50 days) (95\% CI 5.87-28.03) than in (AMWT) patients (88 days) (95\% CI $0.04-0.17)$; $(\mathrm{P}=0.0001)$.

The mean duration of therapy for (NPWT) was (55.5 \pm 21.89 days) versus ( $81.84 \pm 19.76$ days) for (AMWT). Patients were treated in both hospital and home care settings.

With respect to pain scores, both groups showed a significant decrease at the end of follow-up. Comparison of pain scores revealed that they were initially similar during the first weeks of treatment. From week 5 onwards, however, pain scores were significantly lower in the (NPWT) group.

Bacterial Load: We observed that wounds of (NPWT) group showed rapid clearance of bacterial load as compared to (AMWT) group. This was suggested by (40\%) of the cultures in (NPWT) group having no growth by the end of $4^{\text {th }}$ week as compared to $(20 \%)$ in (AMWT) group. Staphylococcus aureus was found to be the most prominent organism in patients of (NPWT) group whereas cultures from (AMWT) group mostly showed mixed growth with Klebsiella, Escherichia Coli and Pseudomonas.

Safety Analysis (Secondary outcomes): Table (4) reports treatment-related rates for 

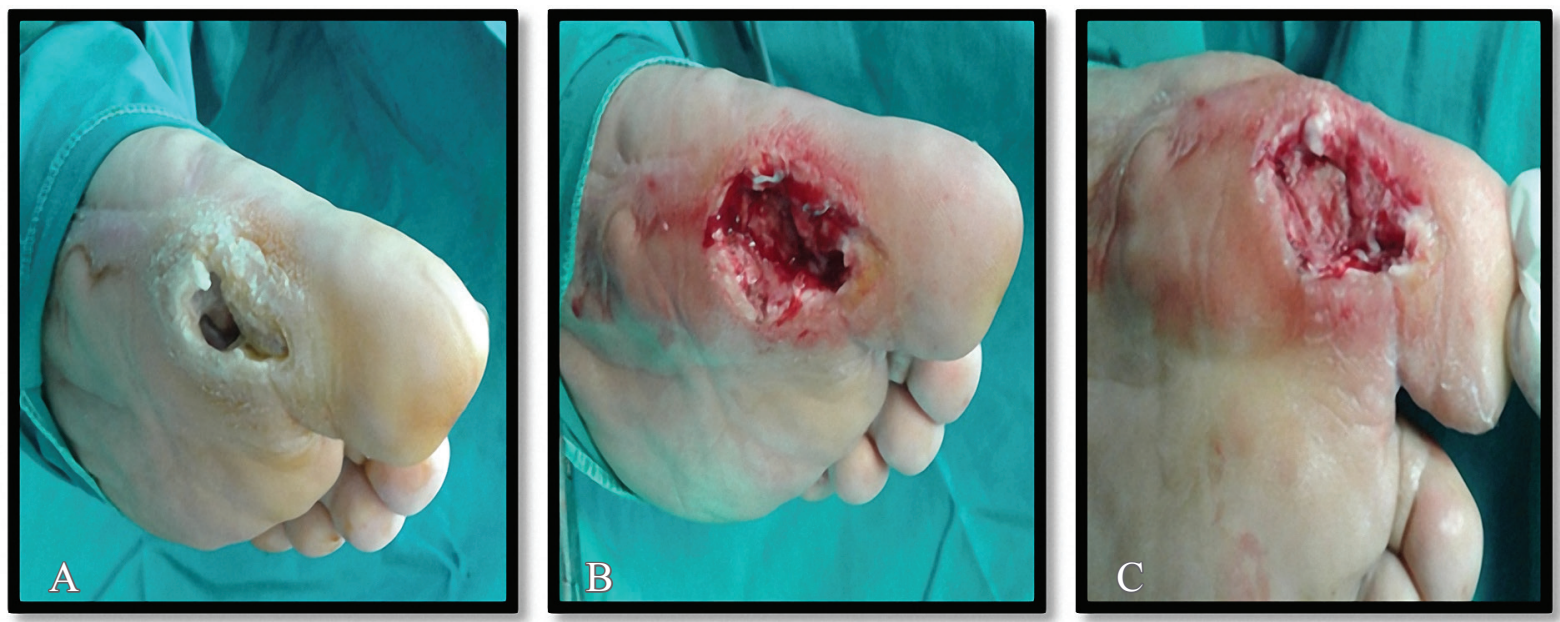

Figure (1): A) Neuropathic foot ulcer. B) After debridement c) One week after VAC dressing application

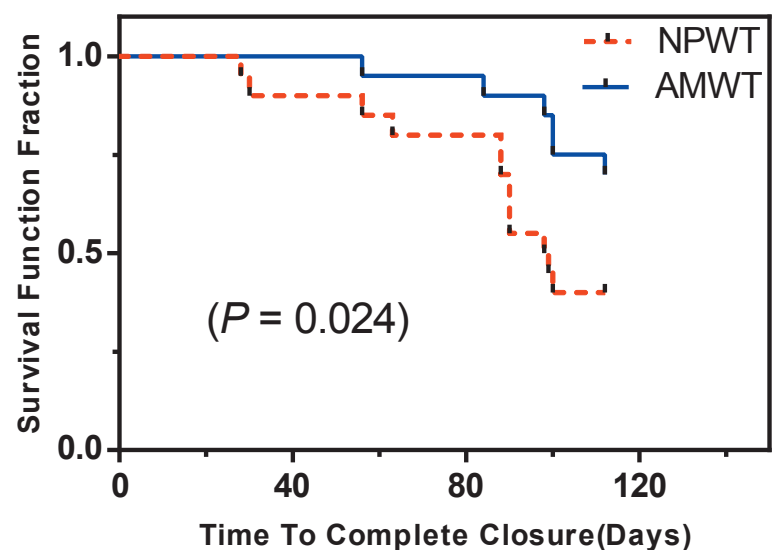

Figure (2): Kaplan-Meier estimates for time to complete wound closure.

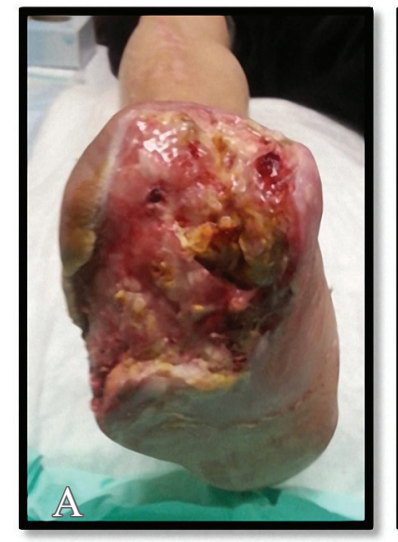

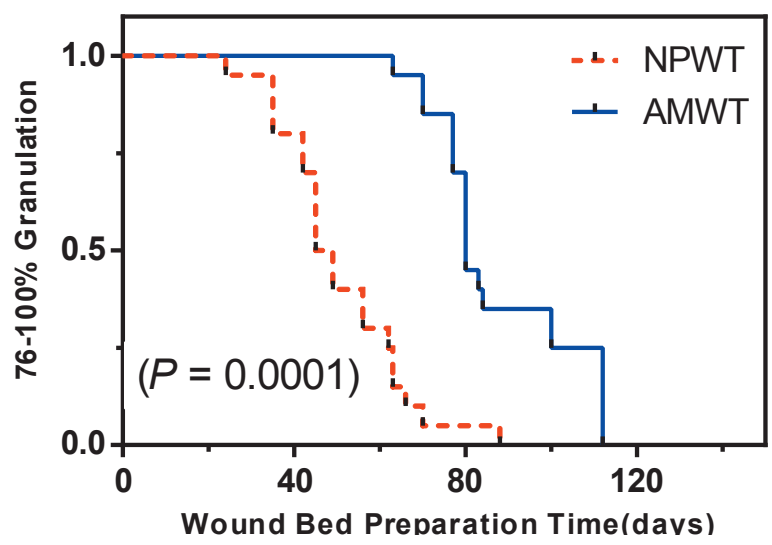

Figure (3): Kaplan-Meier estimates for time to $76-100 \%$ wound granulation.

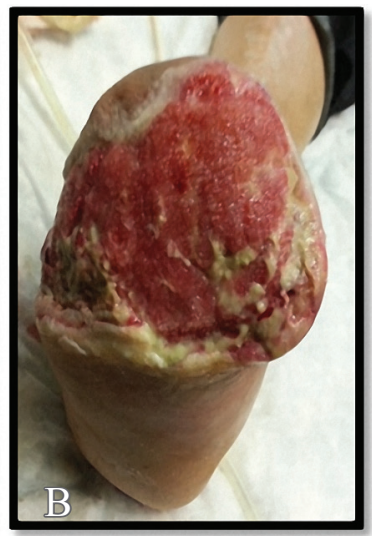

Figure (4): A) Diabetic forefoot amputation stump. B) 8-weeks post VAC application, granulation reached more than $75 \%$.

secondary amputations, edema, cellulitis and wound infection at the end of 16 weeks. Nonsignificantly $(\mathrm{P}=0.42)$ fewer amputations were observed in (NPWT) patients (2 of 20, 10\%) compared with (AMWT) patients
(4 of 20,20\%). The majority of these amputations (2 and 3 , respectively) were minor amputations. In all other categories, no significant differences were observed. 
Table (1): University of Texas Wound Classification System:12.

\begin{tabular}{|c|l|l|l|}
\hline Stages & \multicolumn{1}{|c|}{ Description } & Grading & \multicolumn{1}{c|}{ Description } \\
\hline Stage A & No infection or ischemia & Grade 0 & Epithelialized wound \\
\hline Stage B & Infection present & Grade 1 & Superficial wound \\
\hline Stage C & Ischemia present & Grade 2 & Wound penetrates to tendon or capsule \\
\hline Stage D & Infection and ischemia present & Grade 3 & Wound penetrates to bone or joint \\
\hline
\end{tabular}

Table (2): Patients> and Wounds> Characteristics:

\begin{tabular}{|l|l|l|l|}
\hline \multicolumn{1}{|c|}{ Variable } & NPWT (n=20) & AMWT (n=20) & P-value \\
\hline Age (years) & $55.2 \pm 7.8$ & $53.50 \pm 8.1$ & 0.13 \\
\hline Male & $11 / 20(55 \%)$ & $13 / 20(65 \%)$ & 0.76 \\
\hline Mean ABI & 0.91 & 0.89 & 0.79 \\
\hline Type II Diabetes Mellitus & $19(95 \%)$ & $18(90 \%)$ & 0.94 \\
\hline Smoking & $9(45 \%)$ & $6(30 \%)$ & 0.53 \\
\hline Immobility & $2(10 \%)$ & $1(5 \%)$ & 0.72 \\
\hline Cardiac diseases & $6(30 \%)$ & $4(20 \%)$ & 0.36 \\
\hline Baseline wound duration (weeks) & $7.9 \pm 3$ & $8 \pm 3.13$ & 0.84 \\
\hline Baseline wound area $\left(\mathrm{cm}^{2}\right)$ & $26.54 \pm 14.6$ & $24 \pm 11.4$ & 0.05 \\
\hline
\end{tabular}

Table (3): Wound bed preparation and healing:

\begin{tabular}{|l|l|l|l|}
\hline \multicolumn{1}{|c|}{ Wound Area $\left(\mathbf{c m}^{2}\right)$} & \multicolumn{1}{|c|}{ NPWT } & \multicolumn{1}{|c|}{ AMWT } & P-value \\
\hline Initial & 26.5 & 24 & 0.05 \\
\hline After 4 weeks & 20.3 & 20.1 & 0.82 \\
\hline Final & 3.6 & 5.82 & $0.016^{*}$ \\
\hline Change in wound area & \multicolumn{5}{|l}{} \\
\hline After 4 weeks(cm2)(\%) & $-6.2(23.4)$ & $-3.9(16.25)$ & $0.021^{*}$ \\
\hline Total change(cm2) (\%) & $-22.9(86.4)$ & $-18.18(75.7)$ & $0.002^{*}$ \\
\hline Wound Granulation \% & 11.35 & 13.1 & 0.15 \\
\hline Initial & 47.85 & 28.9 & $0.0001^{*}$ \\
\hline After 4 weeks & 95.65 & 83.1 & $0.0001^{*}$ \\
\hline Final &
\end{tabular}

Table(4): Secondary outcomes of therapy

\begin{tabular}{|l|l|l|l|}
\hline \multicolumn{1}{|c|}{ Variable } & NPWT $(\mathbf{n = 2 0})$ & AMWT $(\mathbf{n}=\mathbf{2 0})$ & P-value \\
\hline Secondary amputation & $2(10 \%)$ & $4(20 \%)$ & 0.42 \\
\hline Edema & $4(20 \%)$ & $3(15 \%)$ & 0.56 \\
\hline Cellulitis & $2(10 \%)$ & $1(5 \%)$ & 0.72 \\
\hline Wound infection & $2(10 \%)$ & $1(5 \%)$ & 0.72 \\
\hline
\end{tabular}

\section{Discussion:}

The present study measures the time in reaching (76-100\%) granulation of open wounds of the foot in diabetic patients. The results demonstrated that the (NPWT) is more effective than conventional treatment in wound bed preparation as it significantly reduced the time to reach (76-100\%) 
granulation up to $43 \%$. Analysis significantly $(\mathrm{P}=0.033)$ demonstrated that (NPWT) resulted in a greater percentage of complete wound healing as well as achievement of $(75 \%)$ wound healing than (AMWT). Moreover, (NPWT) seems to be as safe as the conventional treatments regarding adverse effects, amputations and surgical asepsis.

Because ambulatory (VAC) pump units are available, this treatment could be offered on an outpatient basis. Thus hospitalization days, frequency of dressing changes as well as the number and complexity of required procedures to achieve healing of these wounds would be reduced. Apelqvist et al. ${ }^{14}$ reported a beneficial effect in terms of direct economic cost and resource utilization in patients treated with (VAC) compared to standard moist wound therapy.

Our study included 40 patients into two even groups, their demographic characteristics were comparable with no significant difference between them. Mean age was comparable to a previous large randomized controlled trial ( $\mathrm{RCT}$ ), enrolling 342 patients, having a mean age of 58 years. ${ }^{15}$

Debridement is a key component of healing initiation because it enables removal of devitalized and necrotic tissue that have been shown to retard healing. ${ }^{16}$ NPWT and other wound healing therapies work as adjunctive to debridement. ${ }^{17}$ In the present study, all wounds were debrided then surface areas were measured. Prior to treatment, the mean wound surface areas were $\left(26.5 \mathrm{~cm}^{2}\right.$ vs. $24 \mathrm{~cm}^{2}$ ) in (NPWT vs. AMWT) groups respectively. After 4-weeks, (NPWT) achieved (7.15\%) significantly higher reduction in mean wound area than (AMWT) $(\mathrm{P}=0.021)$. More difference in area reduction was observed at the end of study (112-days), $(10.7 \%)(P=0.002)$. These results correlate well with those obtained by several authors who reported improved healing power on using (NPWT); ${ }^{15,18}$ Blume et al. ${ }^{15}$ found a significant mean area reduction difference of $(9 \%)$ on day $28(\mathrm{P}=0.021)$ favoring (NPWT) over (AMWT). Similarly, Eginton et al. ${ }^{18}$ reported a $(49 \%)$ and $(59 \%)$ reduction in the wound depth and volume, respectively, of 6
(VAC)-treated diabetic foot ulcers. This was significantly greater than the $(7.7 \%)$ reduction in wound depth and $(8 \%)$ reduction in wound volume achieved when the same wounds were treated with moist gauze dressings.

The success of (NPWT) in chronic wounds is associated with removal of excess interstitial fluid, an increase in vascularity, a decrease of bacterial burden and stimulation of granulation tissue formation through the response of wound tissue to the mechanical forces exerted by the application of negative pressure. 19

In this study, (30\%) more (NPWT) patients achieved complete wound healing in 15 days less median time to closure than (AMWT) patients. Thus, (NPWT) hastened complete wound healing by (16.5\%) over (AMWT). This result parallels the findings by several authors. ${ }^{15,19,20}$

In the RCT by Vuerestaek et $\mathrm{al}^{20}$ the median total healing time was (16) days less in the (NPWT) group than the modern wound therapy (hydrogels and alginates) group $(\mathrm{P}=0.0001)$. Ninety percent of the ulcers treated with (NPWT) healed within (43) days versus only (48\%) in the control group. Blume et al, ${ }^{15}$ reported that (14.3\%) more (NPWT) patients achieved complete ulcer closure in less median time than (AMWT) patients.

(NPWT) has been shown to reduce wound dimensions potentially leading to reduced complexity of surgical closure ${ }^{21}$ and to act as a 'bridge' to manage the wound until surgical closure is possible. ${ }^{22}$ Formation of granulation tissue is a part of the proliferative stage of wound healing. A freshly granulating wound surface indicates good healing. ${ }^{17}$ Time from therapy initiation to achievement of a continuous fresh bed of granulation in the wound was taken as time needed for wound bed preparation for further surgical closure. Saxena et al. ${ }^{17}$ and Greene et al. ${ }^{23}$ have elucidated the role of open pore foam dressing in the creation of micromechanical deformations of the wound surface. These micromechanical deformations are caused when negative pressure draws tissue into the foam pores. This stretches cells and promotes cell division that stimulates granulation tissue 
formation. ${ }^{17}$

In the present study, the median time needed to achieve (76-100\%) granulation tissue formation was significantly shorter in (NPWT) patients (50 days) than in (AMWT) patients (88 days) $(\mathrm{P}=0.0001)$. Several authors had demonstrated the role of (NPWT) in initiation and improvement of granulation tissue formation. Morykwas et al. ${ }^{24}$ demonstrated that wounds treated with negative pressure achieved more granulation using either continuous or intermittent pressure than those treated using conventional dressing. Similarly, in another study by Armstrong and Lavery, ${ }^{25}$ the rate of (76-100\%) granulation tissue formation was significantly faster in the (NPWT) group than in the control group $(\mathrm{P}=0.002)$. In a larger RCT by Blume et al. ${ }^{15}$ that included (342) diabetic patients, (46) patients presented with $(0-10 \%)$ granulation at baseline inclusion $(24$ NPWT and 22 AMWT). Of these, (70.8\%) of (NPWT) patients and (36.4\%) of (AMWT) patients achieved (76-100\%) granulation $(\mathrm{P}=0.019)$. Median time for $(76-100 \%)$ granulation tissue formation was significantly shorter for (NPWT); (56 days) than for (AMWT); (114 days) $(\mathrm{P}=0.022)$.

An additional benefit observed was the ability of (VAC) therapy to alleviate bacterial infection in a wound. In the study performed by Morykwas et al. ${ }^{24}$ (VAC) therapy achieved a significant reduction in bacterial load of chronic wounds by the fifth day. A similar reduction, however, took (11 days) in control wounds. We observed that wounds of (NPWT) group showed faster clearance of bacterial load as compared to (AMWT) group; suggested by (20\%) more negative cultures in (NPWT) group by the 4th week. Our study correlates with the study by Moues et al. ${ }^{26}$ who had observed that nonfermentative Gram-negative bacilli showed a significant decrease whereas S. Aureus showed an increase in (VAC)-treated wounds.

Safety analysis demonstrated that (NPWT) is as safe as (AMWT) in the treatment of diabetic foot wounds. No significant difference was observed in wound-related complications such as infection, edema, cellulitis and amputation. Lone et al. ${ }^{27}$ observed the safety of (VAC) over salinemoistened gauze, in terms of fewer numbers of secondary amputations. Blume et al. ${ }^{15}$ also reported fewer number of secondary amputations in (VAC) treated patients as compared to those treated by (AMWT).

When making a decision to stop (NPWT), it must be determined whether the initial objectives of therapy have been achieved. If so, it must be considered whether any further benefit can be achieved through the continued use of (NPWT), or whether transfer to an alternative method of wound management may be more appropriate. ${ }^{28}$

At present, the concept of keeping a humid environment to favor the closure of wounds is widely known and accepted. ${ }^{29}$ But it seems, keeping the wound humid is not enough, other elements are required to implement a comprehensive management plan that includes sufficient debridement, effective offloading of plantar area, tight glycemic control, revascularization if indicated in addition to advanced methods of local wound care as (NPWT). ${ }^{30}$

In summary, our data demonstrates that negative pressure wound dressings decrease the wound size more effectively than advanced moist wound dressings as hydrogels and alginates. It is suggested that (NPWT) is a safe, easy to use and patientfriendly method of treating diabetic foot wounds which helps in early closure of wounds, preventing complications and hence promising a better outcome.

\section{Conclusion:}

NPWT is as safe as and more efficacious than AMWT for the treatment of diabetic foot wounds.

\section{Reference:}

1- Falanga V: Classifications for wound bed preparation and stimulation of chronic wounds. Wound Repair Regen 2000; 8: 347-352.

2- Lazarus GS, Cooper DM, Knighton DR: Definitions and guidelines for assessment of wounds and evaluation of healing. Arch Dermatol 1994; 130(4): 489-493. 
3- Mustoe TA, O'Shaughnessy K, Kloeters O: Chronic wound pathogenesis and current treatment strategies: A unifying hypothesis. Plast Reconstr Surg 2006; 117(Supp17): $35 \mathrm{~S}-41 \mathrm{~S}$.

4- Marston WA: Dermagraft Diabetic Foot Ulcer Study Group. Risk factors associated with healing chronic diabetic foot ulcers: The importance of hyperglycemia. Ostomy Wound Manage 2006; 52: 26-28.

5- Brem H, Sheehan P, Rosenberg HJ, Schneider JS, Boulton AJ: Evidence-based protocol for diabetic foot ulcers. Plast Reconstr Surg 2006; 117(Supp17): S193-S211.

6- Wu C, Marston W, Armstrong DG: Wound care: The role of advanced wound healing technologies. J Vasc Surg 2010; 52: 59S-66S.

7- Nain SP, Uppal KS, Garg R, Bajaj K, Garg SH: Role of negative pressure wound therapy in healing of diabetic foot ulcers. $J$ Surg Tech Case Rep 2011; 3: 17-22.

8- Joseph E, Hamori CA, Bergman S, Roaf E, Swann NF, Anastasi GW: A prospective, randomized trial of vacuum assisted closure versus standard therapy of chronic non healing wounds. Wounds 2000; 12: 60-67.

9- Philbeck TE, Schroeder WJ, Whittington KT: Vacuum-assisted closure therapy for diabetic foot ulcers: Clinical and cost analysis. Home Health Consult 2001; 8: 26-34.

10- Schwien T, Gilbert J, Lang C: Pressure ulcer prevalence and the role of negative pressure wound therapy in home health quality outcomes. Ostomy Wound Manage 2005; 51: 47-60.

11- Moisidis E, Heath T, Boorer C, Ho K, Deva AK: A prospective, blinded, randomized, controlled clinical trial of topical negative pressure use in skin grafting. Plast Reconstr Surg 2004; 114: 917-22.

12- Oyibo SO, Jude EB, Tarawneh I, Nguyen HC, Harkless LB, Boulton AJ: A comparison of two diabetic foot ulcer classification systems: The Wagner and the University of Texas wound classification systems. Diabetes Care 2001; 24: 84-88.

13- Xakellis GC Jr, Frantz RA: Pressure ulcer healing: What is it? What influences it? How is it measured? Adv Wound Care 1997; 10(5): 20-26.

14- Apelqvist J, Armstrong DG, Lavery LA, Boulton AJ: Resource utilization and economic costs of care based on a randomized trial of vacuum-assisted closure therapy in the treatment of diabetic foot wounds. Am J Surg 2008; 195: 782-788.
15- Blume PA, Walters J, Payne W, Ayala J, Lantis J: Comparison of negative pressure wound therapy using vacuum-assisted closure with advanced moist wound therapy in the treatment of diabetic foot ulcers: A multicenter randomized controlled trial. Diabetes Care 2008; 31: 631-636.

16- Etoz A: Negative pressure wound therapy on diabetic foot ulcers. Wounds 2007; 19(9): 250-254.

17- Saxena V, Hwang C, Huang S, Eichbaum Q, Ingber D, Orgill DP: Vacuum assisted closure: Microdeformations of wounds and cell proliferation. Plast Reconstr Surg 2004; 114: 1086-1096.

18- Eginton MT, Brown KR, Seabrook GR, Towne JB, Cambria RA: A prospective randomised evaluation of negative-pressure wound dressings for diabetic foot wounds. Ann Vasc Surg 2003; 17: 645-649.

19- Argenta LC, Morykwas MJ: Vacuumassisted closure: A new method for wound control and treatment: Clinical experience. Ann Plast Surg 1997; 38: 563-576.

20- Vuerstaek J, Vainas T, Wuite J, Nelemans P, Neumann M, Veraart J: State-of-the-art treatment of chronic leg ulcers: A randomized controlled trial comparing vacuum-assisted closure (V.A.C.) with modern wound dressings. J Vasc Surg 2006; 3, 122-128.

21- Bollero D, Carnino R, Risso D, Gangemi E, Stella M: Acute complex traumas of the lower limbs: A modern reconstructive approach with negative pressure therapy. Wound Repair Regen 2007; 15(4): 589-594.

22- Rinker B, Amspacher J, Wilson PC, Vasconez HC. Subatmospheric pressure dressing as a bridge to free tissue transfer in the treatment of open tibia fractures. Plast Reconstr Surg 2008; 121(5): 1664-1673.

23- Greene AK, Puder M, Roy R, Arsenault D, Kwei S, Moses MA, Orgill DP: Microdeformational wound therapy: Effects on angiogenesis and matrix metalloproteinases in chronic wounds of 3 debilitated patients. Ann Plast Surg 2006; 56: 418-422.

24- Morykwas MJ, Argenta LC, Shelton-Brown EI, McGuirt W: Vacuum assisted closure: A new method for wound control and treatment: Animal studies and basic foundation. Ann Plast Surg 1997; 38: 553-562.

25- Armstrong DG, Lavery LA: Negative pressure wound therapy after partial diabetic foot amputation: A multicentre, randomised controlled trial. Lancet 2005; 
366: 1704-1710.

26- Moues CM, Vos MC, van den Bemd GJ, Stijnen T, Hovius SE: Bacterial load in relation to vacuum-assisted closure wound therapy: A prospective randomized trial. Wound Repair Regen 2004; 12: 11-17.

27- Lone A, Zaroo M, Laway B, Pala N, Bashir SH, Rasool A: Vacuum-assisted closure versus conventional dressings in the management of diabetic foot ulcers: A prospective casecontrol study. Diabetic Foot \& Ankle 2014; 5: 233-245.

28- Dunn R, Hurd Th, Chadwick P, Cote J, Cockwill J, Mole T, Smith J. Factors associated with positive outcomes in 131 patients treated with gauze-based negative pressure wound therapy. Intern J Surg 2011; (9) 258-262.

29- Sepúlveda G, Espíndola M, Maureira M, Sepúlveda E, Ignacio J, Oliva C, Sanhueza A, Vial M, Manterola C: Negative-pressure wound therapy versus standard wound dressing in the treatment of diabetic foot amputation. A randomised controlled trial. CIR ESP 2009; 86(3): 171-177.

30- Guffanti A: Negative pressure wound therapy in the treatment of diabetic foot ulcers. A systematic review of the literature. $J$ Wound, Ostomy Continence Nurs 2014; 41(3): 233-237. 